\title{
A UNIQUE SYMBIOTIC-LIKE/CATACLYSMIC TRIPLE STAR 4 DRA (=CQ DRA): SIX YEARS OF UBV MONITORING
}

\author{
Z. URBAN ${ }^{1}$, L. HRIC ${ }^{1}$, L. LEEDJ $\ddot{A R V}{ }^{2}$ \\ 1. Astronomical Institute of the Slovak Academy of Sciences, \\ 05960 Tatranská Lomnica, Slovakia \\ e-mail: urban@ta3.sk, hric@ta3.sk \\ 2. Tartu Astrophysical Observatory, 2444 Tóravere, Estonia \\ e-mail: leed@jupiter.aai.ee
}

The bright $\left(m_{\mathbf{v}} \approx 5\right)$ M3III star 4 Dra (HD108907, HR4765, CQ Dra) is a unique triple system combining in itself the basic structural properties of a symbiotic-like binary and of a cataclysmic one. The red giant 4 Dra A forms a wide binary ( $\mathrm{P}_{\text {orb }} \approx 1703 \mathrm{~d}$ ) with a hot companion 4 Dra $\mathrm{B}$ which seems to be a compact cataclysmic binary system (4 Dra BC, probable $\mathrm{P}_{\text {orb }}$ of about $4 \mathrm{~h}$ ).

We have monitored the 4 Dra system in $U B V$ at the Skalnaté Pleso and Stará Lesná Observatories and at the Tartu Astrophysical Observatory during a total of 205 nights between 1989 March 12 and 1995 June 20; a few additional data points were obtained at the Bucharest Observatory.

Our long-term $U B V$ light curves (LCs) show:

- The modulation of the $U$ brightness with the $1703 \mathrm{~d}$ period.

- Two sudden $U$ brightenings, centered at phases 0.1 and 0.0 , respectively, with similar amplitudes of about 0.3 mag but of apparently different duration, their peak-to-peak separation being about $1583 \mathrm{~d}$.

- Pulsation-like cycles well-correlated in all three colours, superimposed in the $U$ on the long-term orbital modulation, with mean period of 340 $\pm 10 \mathrm{~d}$ (almost exactly one fifth of the 1703-d period).

- Irregular variations in all three colours, with an amplitude of 0.1-0.2 mag, often simultaneous or quasi-simultaneous, of similar brightnesschange profiles and typical time-scale of tens of days.

- Two specific intervals (so-called plateaux) in the $B$ and $V$ LCs where the level of the irregular variability appears to be supressed. They display a mild steady brightness increase. Both are located near the periastron and thus correlated with the sudden brightenings in $U$.

- The globally minimal values in $U, B$ and $V$ occur near phase 0.5 . 
The phenomenological model of the wide 4 Dra $A+B C$ system we use is based on the published spectroscopic orbital solution and our derivation of the corresponding Roche geometry. Although the red giant star seems to be well inside its Roche lobe, given the extended and unstable atmospheres of red giants and the observed intense mass loss via stellar winds from these stars, almost all of its critical Roche volume could be filled by gas of various density and temperature. This could occur especially during the periastron passage time when some kind of intense tidal interaction could still occur, despite the rather large separation of 4 Dra A and 4 Dra BC even at that time. A stimulated mass loss from the red giant's extended amosphere towards the hot component, or some collimation of the outflowing stellar wind at least, seems to be quite plausible then. We thus conclude that, as well as being an, as yet, unique testing site for the evaluation of the possible parameters of the precursor systems to cataclysmic variables, 4 Dra $\mathrm{A}+\mathrm{BC}$ provides us also with an equally unique environment for studying the modulated wind accretion by a cataclysmic binary.

We attribute the long-term modulation of the $U$ LC of the 4 Dra $\mathrm{A}+\mathrm{BC}$ system to some variety of reflection effect. Near-periastron brightenings in the $U$ could be ascribed either to the Rayleigh scattering of the light coming from $4 \mathrm{Dra} \mathrm{BC}$ in the red giant's amosphere, or to the irradiation of a cloud enveloping 4 Dra $\mathrm{BC}$ as a result of elevated mass influx during the near-periastron stage by a cataclysmic binary inside it, or perhaps even to a direct response of this cataclysmic binary to an increased wind accretion. The interval of about $1583 \mathrm{~d}$ between the two events, different from the radial-velocity-determined orbital period of $1703 \mathrm{~d}$, suggests rather a physical dynamical interpretation than some kind of an 'orbital clock'. The pulsation-like cycles are red giant pulsations. The irregular light variations reflect some processes within the extended atmosphere of the red giant component. Their conspicuous supression near the periastron suggests invisibility of the relevant source region or perhaps a kind of switch-off or masking of the parent mechanisms by the dynamical processes occurring at this stage. The global $U B V$ minimum at about phase 0.5 corresponds to a partial eclipse of the reflection effect region by 4 Dra BC and its circumsystem material.

We refer the interested reader to the full-length version of this paper (to be submitted to Astron. and Astrophys.) for all the details and references. Acknowledgements. We thank D. Reimers for communicating his results to us prior to publication, and R.F. Griffin for a consultation. 